\title{
Highly Deformable Thermal Interface Materials Enabled By Covalently-Bonded Carbon Nanotubes
}

\author{
Hong Wang ${ }^{1}$, Abdullah S Tazebay ${ }^{1}$, Gang Yang ${ }^{1}$, Henry Lin², Woongchul Choi ${ }^{2}$, \\ Choongho $\mathrm{Yu}^{1,2 *}$ \\ ${ }^{1}$ Department of Mechanical Engineering \\ ${ }^{2}$ Department of Materials Science and Engineering \\ Texas A\&M University \\ College station, Texas 77843 USA
}

* Corresponding author. Tel:+1 979 862-1073. E-mail: chyu@tamu.edu (Choongho Yu) 


\begin{abstract}
The exceptional thermal conductivity of individual carbon nanotubes have rarely materialized in bulk materials mainly due to the large thermal contact resistance between carbon nanotubes (CNTs). This can be attributed to weak van der Waals bonding at the CNT junctions where the outstanding phonon transport along the strong covalent bonding on the graphitic layer is largely impeded. In bulk materials, however, it has been extremely difficult to achieve covalently bonded junctions between CNTs. Here we report polymer composites consisting of sponge-like CNT structures whose junctions between CNTs are covalently bonded, resulting in a high thermal conductivity and a low Young's modulus, which are hard to achieve at the same time. The low modulus allows thermal interface material (TIM) to easily deform to make the surfaces of heat sink/source fully in contact, which is essential for TIM. Our facile scalable preparation process also makes our composite very attractive as TIM as well as provides insight to better utilize high thermal conductivity of CNTs.
\end{abstract}




\section{Introduction}

Thermal interface material (TIM) is widely used to effectively transfer thermal energy from power consuming devices to heat sinks. When two rigid materials are in contact, voids created by surface asperity cause poor heat transfer.[1] Such inefficient heat transfer from heat sources to heat sinks has been well-known crucial issues in thermal management, which now becomes much more important due to highly integrated modern devices. Recent performance improvement in the thermal conductivity of TIM made it possible to achieve much higher values from $\sim 0.4 \mathrm{~W} / \mathrm{m}-\mathrm{K}$ of popular commercial TIMs.[2, 3] However, their mechanical properties are largely unknown or improper although making TIM soft enough to effectively fill the voids is imperative for practical use.

A common strategy to make TIM is to add fillers of high thermal conductivity to a polymer matrix. Carbon nanotube $(\mathrm{CNT})$ is a popular filler material owing to the high thermal conductivity $\left(\sim 10^{3} \mathrm{~W} / \mathrm{m}-\mathrm{K}\right)$ of "individual” CNTs.[4] Nevertheless, CNT-polymer composites often have thermal conductivities lower than $1 \mathrm{~W} / \mathrm{m}-\mathrm{K}[5-8]$ even with high CNT concentrations. It is because that the macroscopic thermal conduction in CNT-filled polymer composites is governed by the thermal contact resistance at the interface at CNT-CNT junctions rather than the intrinsic thermal conductivity of individual CNTs.[9-12] This poor thermal conduction can be ascribed to the weak van der Waals interaction at the junction, so covalently-bonded junctions in CNT networks are expected to improve heat transport at the junction.[13-16] However, this has been only feasible theoretically or experimentally for a single junction at a time using electron beam, which is hard to be utilized in practically viable bulk composites. 
Due to the difficulty of strengthening the bonding at CNT junctions, there have been efforts to make vertically aligned CNTs to increase the thermal conductivity of CNT-polymer composites along the axial direction of CNT. For instance, $1.4 \mathrm{~W} / \mathrm{m}-\mathrm{K}$ at room temperature was reported for aligned CNT-epoxy composites prepared using a microwave-assisted method.[17] Higher thermal conductivity up to $4.9 \mathrm{~W} / \mathrm{m}-\mathrm{K}$ was reported for CNT-polymer composites with densely-packed aligned CNT.[18] However, these aligned CNTs often suffer from low deformability, making poor thermal contacts with heat sinks/sources. The alignment of CNTs also requires significant efforts, which may not be suitable for a scale-up process.[19]

Other fillers such as graphene,[20] ceramics,[21] and metals[22] have also been studied but heat flow in these materials are still limited by the thermal contact resistance between the fillers. High concentrations of inorganic fillers make composites unmalleable and brittle. The thermal conductivity of graphene is preferentially high along the in-plane direction, so significant efforts are required to orient graphene plane along the thickness direction of TIM. Graphene also requires long processing time for dispersion and purification/centrifugation. To date, elastomeric TIMs with scalability and decent thermal conductivity are scarcely reported.

Here we report highly deformable sponge-like CNT based TIM prepared by a scalable and facile method by infiltrating polyvinylidene fluoride (PVDF) into three-dimensional (3D) sponge-like CNT bulks. Our method is different from the typical TIM preparation methods mixing fillers with polymers to make slurry. The CNT sponges have branched network structures with covalently-joined junctions to ensure good thermal transport along the CNT structure as well as maintain a large deformability without fracture. 


\section{Results and Discussion}

CNT sponges were bulk-produced in a 3-zone furnace tube whose inner diameter is $22 \mathrm{~mm}$ without using any substrates (see sliced CNT sponges in Fig. 1a).[23] We synthesized two different types of CNT sponges - one kind mainly consists of covalently bonded tubular structures (labeled as c-CNT) and the other kind contains spherical C-Fe particles (labeled as p-CNT). The well connected graphitic tubular structures in c-CNT are excellent pathways for heat transport (Fig. 1b, c, d, e, and f). The p-CNT sponges were made to have comparative studies for investigating the influence of disengaged particles on thermal conductivity (Fig. 1g), which mimics a case of typical commercial TIMs containing spherical particles in a polymer matrix. The disengaged particles often suffer from large thermal contact resistance between the conductive additives and polymer matrix.

In a typical 30-min synthesis to obtain c-CNT sponges whose diameter is $\sim 22 \mathrm{~mm}$ and length is $\sim 100 \mathrm{~mm}, \sim 0.3$-g ferrocene (Sigma-Aldrich, 98\%) was placed in the first upstream zone $\left(120^{\circ} \mathrm{C}\right)$ to deliver iron as a CNT catalyst to the last zone $\left(650^{\circ} \mathrm{C}\right)$ where CNTs grew under the flow of hydrogen (260 sccm, Airgas, 99.999\%) and ethylene (80 sccm, Airgas, 99.999\%) gases together with argon (80 sccm, Airgas, 99.999\%) gas passed through a water bubbler. Note that ferrocence decomposes into iron and other compounds at the reaction zone.[23] We used a lower reaction temperature $\left(600{ }^{\circ} \mathrm{C}\right)$ to obtain p-CNT sponges. Our method is simple and scalable, and synthesis cost is only $\sim \$ 0.1$ per gram, which is much lower than that for aligned CNT.[23] 

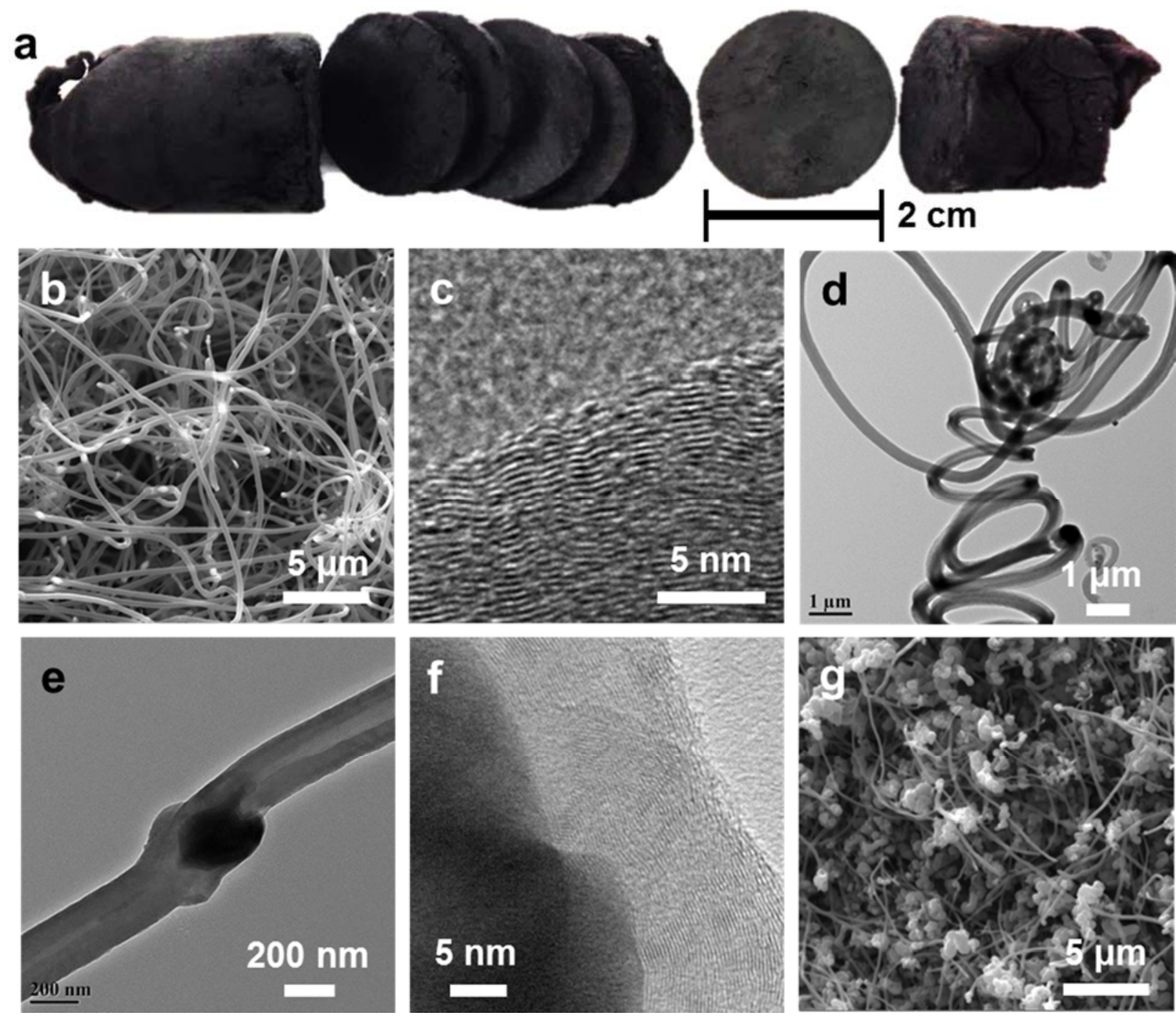

Figure 1. (a) Photograph of as synthesized cylindrical CNT sponge which was sliced into small disks with a diameter of $\sim 2.2 \mathrm{~cm}$. (b) SEM image of c-CNT sponges whose density is 40 50 $\mathrm{mg} / \mathrm{cm}^{3}$. (c) TEM image of a c-CNT sponge. (d) SEM image of covalently bonded junctions in a c-CNT sponge. (e) TEM image of a covalently bonded junction. (f) TEM image showing graphitic layers near a covalently bonded junction. (g) SEM image of a p-CNT sponge whose density is $80 \sim 100 \mathrm{mg} / \mathrm{cm}^{3}$.

Scanning electron microscope (SEM) images of an as-synthesized CNT sponge show long CNTs were joined and intertwined, as shown in Fig. 1b. The CNTs typically have diameters of 
$200 \sim 400 \mathrm{~nm}$ and lengths of $30 \sim 50 \mu \mathrm{m}$. The graphitic layers shown in the transmission electron microscope (TEM) image (Fig. 1c) confirm multi-wall CNTs. Figure 1d displays several CNTs were joined, and a close-up view of a junction (Fig. 1e) shows two CNTs connected to a catalyst particle at the center. The graphitic layers near the catalysts and the CNTs shown in Fig. S1a,b and Fig. 1f (see an enlarged image shown in Fig. S1c) suggests CNTs were covalently bonded. The p-CNT sponges contained amorphous carbon/catalyst (spherical particles) along with CNTs (Fig. 1g, Fig. S1e,f). The particles in p-CNT are composed of carbon and iron compounds (Fig. S1g). The diameter and length of CNTs in p-CNT are slightly smaller than those of c-CNT due to the lower growth temperature.

To infiltrate a PVDF solution into the CNT sponges, the sponges were sliced into a coin shape with thicknesses of $3 \sim 6 \mathrm{~mm}$, and then treated with ozone plasma (UV/Ozone procleaner, Bioforce nanosciences) for $1 \mathrm{~h}$ to make the CNT surface more hydrophilic. It was found that ozone plasma treatment creates hydroxyl and carboxyl groups on the surface of CNT.[24] The sponge slices were soaked by a solution containing 5-wt\% PVDF (99\%, Alfa Aesar) in dimethylformamide (DMF) (99\%, Alfa Aesar), and then degassed in vacuum ( $\sim .1$ Torr) at room temperature for $30 \mathrm{~min}$ and dried at $160{ }^{\circ} \mathrm{C}$ for $2 \mathrm{~h}$. Figure 2a shows a photo of a PVDF-filled CNT sponge. The CNTs are connected by catalyst particles as schematically illustrated in Fig. 2a, based on multiple SEM images of pristine CNT sponges (see Fig. S2). 

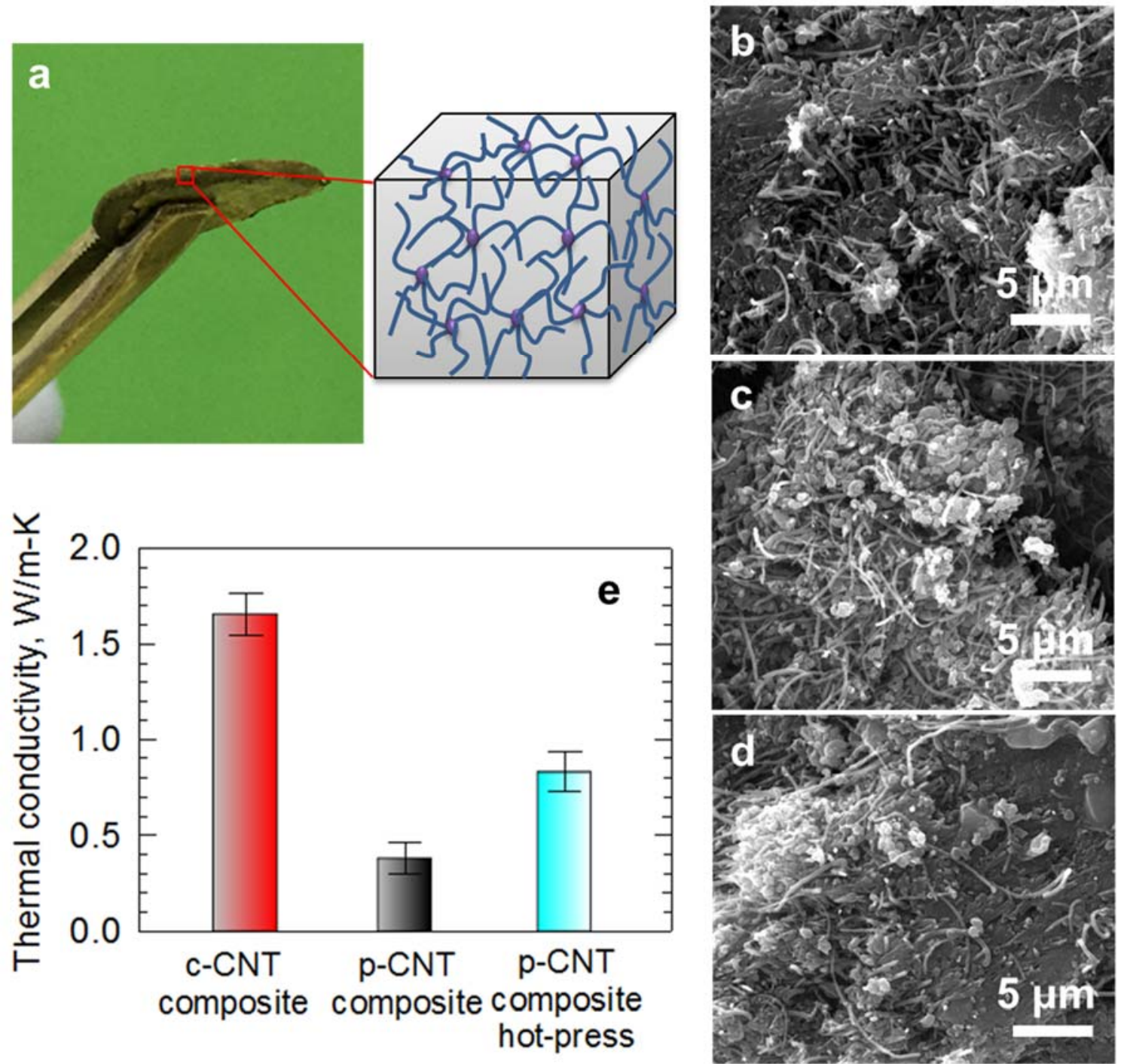

Figure 2. (a) Photograph of a PVDF-filled CNT sponge composite and an illustration of the embedded CNT structure. Purple dots were drawn to indicate covalently bonded junctions and blue curvy lines indicate CNTs. SEM images of a c-CNT sponge composite (b), a p-CNT sponge composite (c), and a p-CNT sponge composite after the hot press process (d). (e) Room temperature thermal conductivities of the c-CNT composite $(21 \mathrm{wt} \% \mathrm{CNT})$ and the p-CNT sponge composite $(22 \mathrm{wt} \% \mathrm{CNT})$ before and after the hot press process. 
The samples were cut along the thickness direction with a razor blade and their cross sections were inspected. Composites with c-CNT (Fig. 2b, Fig. S3a,b) have fewer aggregated particles compared to composites with p-CNT sponge (Fig. 2c, Fig. S3c,d). The void vol\% of the p-CNT sponge composite was suppressed after a hot-press process at $160{ }^{\circ} \mathrm{C}$ and $29 \mathrm{MPa}$ for 60 min, as shown in Fig. 2d and Fig. S3e,f.

The out-of-plane thermal diffusivity of the disc samples (typically $1 \sim 2.5 \mathrm{~mm}$ in thickness) was measured by using Flashline 3000 (TA instruments), and then thermal conductivity $(k)$ was calculated with the formula, $k=c \rho \alpha$, where $c, \rho$, and $\alpha$ are specific heat, mass density, and thermal diffusivity, respectively. We estimated the specific heat of the composites by taking linear averages of those for CNT $(0.76 \mathrm{~J} / \mathrm{g}-\mathrm{K})[25]$ and PVDF (1.6 J/g-K).[26]

The thermal conductivity of the c-CNT sponge composite was measured to be $\sim 1.66 \mathrm{~W} / \mathrm{m}-\mathrm{K}$, which is much higher than $\sim 0.38 \mathrm{~W} / \mathrm{m}-\mathrm{K}$ from the $\mathrm{p}$-CNT composite. It should be noted that the sponge $\mathrm{wt} \%$ for both samples were similar (21 and $22 \mathrm{wt} \%$ ). The relatively low thermal conductivity from the p-CNT composite could be partly caused by a relatively large concentration of pores since the particles impede infiltration of PVDF. After the hot-press process of the $\mathrm{p}$-CNT composite, the thermal conductivity was increased to $0.83 \mathrm{~W} / \mathrm{m}-\mathrm{K}$, but it is still lower than that of the c-CNT composite. Note that all the thermal conductivity values were measured at room temperature.

Here we further analyzed thermal conductivity of the sponge portion so that we identify the effectiveness of covalent bonding in the sponge. To extract thermal conductivity of the sponge, we calculated the volume fraction of voids ( $\left.\phi_{\text {Void }}\right)$ in the composites using experimentally and 
theoretically obtained density values.

$$
\phi_{\text {Void }}=1-\frac{\rho_{\text {Composite (Experimental) }}}{\rho_{\text {Composite (Theoretical) }}}=1-\frac{\rho_{\text {Composite (Experimental) }}}{\rho_{C N T} \gamma_{C N T}+\rho_{P V D F} \gamma_{P V D F}}
$$

where $\gamma$ is the weight fraction of the indexed material. The experimentally obtained density of the composites with c-CNT was $1.29 \mathrm{~g} / \mathrm{cm}^{3}$, and those for $\mathrm{p}-\mathrm{CNT}$ before and after the hot press were respectively $0.46 \mathrm{~g} / \mathrm{cm}^{3}$ and $1.09 \mathrm{~g} / \mathrm{cm}^{3}$. The theoretical density values were estimated using 2.1 $\mathrm{g} / \mathrm{cm}^{3}$ for the density of typical multi-wall CNTs[27] ( PVDF[26] $\left(\rho_{P V D F}\right)$. The void fraction for the composite with c-CNT, was calculated to be 0.3 , and those for p-CNT before and after the hot press were respectively 0.75 and 0.41 .

Thermal conductivity of a composite can be described using a parallel resistor model.[1, 28]

$$
k_{\text {Composite }}=\phi_{C N T} k_{C N T}+\phi_{P V D F} k_{P V D F}+\phi_{V o i d} k_{\text {Void }}
$$

where $k_{\text {Void }} \approx 0$ and $\phi$ is the volume fraction of the indexed material, which can be described using the weight fraction of CNT sponge and PVDF as:

$$
\phi_{C N T \text { (or PVDF) }}=\left[\frac{\gamma_{C N T}\left(\text { or } \gamma_{P V D F}\right)}{\rho_{C N T}}\left(1-\phi_{V o i d}\right)\right] /\left[\frac{\gamma_{C N T}}{\rho_{C N T}}+\frac{\gamma_{P V D F}}{\rho_{P V D F}}\right]
$$

The thermal conductivity of PVDF is assumed to be $0.25 \mathrm{~W} / \mathrm{m}-\mathrm{K}$ according to literature.[29, 30] Then the thermal conductivity of the c-CNT sponge was calculated to be $12 \mathrm{~W} / \mathrm{m}-\mathrm{K}$, which is higher than 6.9 and $6.3 \mathrm{~W} / \mathrm{m}-\mathrm{K}$ from the p-CNT composites before and after the hot-press process, respectively. These values are much higher than $\sim 0.2 \mathrm{~W} / \mathrm{m}-\mathrm{K}$ from CNT films (along the out-of-plane direction) where CNTs were presumably connected by only van der Waals bonding.[31] The spherical particles in p-CNT are estimated to occupy a half of its mass from the density difference between those of the c-CNT sponge prior to PVDF infiltration $\left(40 \sim 50 \mathrm{mg} / \mathrm{cm}^{3}\right)$ 
and p-CNT sponge $\left(80 \sim 100 \mathrm{mg} / \mathrm{cm}^{3}\right)$. This suggests that covalently bonded CNTs are the key to improve thermal conductivity.

Table 1. Thermal conductivity $(k)$ and thermal conductivity enhancement (TCE) of $\mathrm{CNT} /$ polymer composites at room temperature

\begin{tabular}{cccccc}
\hline CNT configuration & $\boldsymbol{k}(\mathbf{W} / \mathbf{m}-\mathbf{K})$ & TCE & Polymer matrix & CNT filler & References \\
\hline c-CNT sponge & 1.66 & $560 \%$ & PVDF & $21 \mathrm{wt} \%$ & This work \\
\hline Randomly & 0.50 & $200 \%$ & PANI & $80 \mathrm{wt} \%$ & $\operatorname{Ref}[32]$ \\
dispersed CNT & 0.39 & $30 \%$ & PEDOT:PSS & $60 \mathrm{wt} \%$ & $\operatorname{Ref}[6]$ \\
& 0.90 & $350 \%$ & Epoxy & $9 \mathrm{vol} \%$ & $\operatorname{Ref}[33]$ \\
& 0.48 & $200 \%$ & Epoxy & $7 \mathrm{wt} \%$ & $\operatorname{Ref}[34]$ \\
& 0.52 & $190 \%$ & Epoxy & $7 \mathrm{vol} \%$ & $\operatorname{Ref}[35]$ \\
& 0.40 & $55 \%$ & PMMA & $7 \mathrm{wt} \%$ & $\operatorname{Ref}[36]$ \\
& 0.30 & $50 \%$ & PS & $1 \mathrm{wt} \%$ & $\operatorname{Ref}[37]$ \\
Aligned CNT & 0.25 & $4 \%$ & Epoxy & $0.5 \mathrm{wt} \%$ & $\operatorname{Ref}[38]$ \\
& 4.80 & $1800 \%$ & Epoxy & $17 \mathrm{vol} \%$ & $\operatorname{Ref}[18]$ \\
& 1.21 & $280 \%$ & Silicone & $3 \mathrm{wt} \%$ & $\operatorname{Ref}[39]$ \\
& 1.40 & $460 \%$ & Epoxy & unknown & $\operatorname{Ref}[17]$ \\
\hline
\end{tabular}

Abbreviation: Poly(3,4-ethylenedioxythiophene)-poly(styrenesulfonate) (PEDOT:PSS); Polyaniline (PANI); Poly(methyl methacrylate) (PMMA); Polystyrene (PS).

Thermal conductivity enhancement was calculated using $\left(k_{\text {Composite }}-k_{P V D F}\right) / k_{P V D F}$. The enhancement of composite with a c-CNT sponge concentration of $21 \mathrm{wt} \%$ was $560 \%$. For comparison, thermal conductivities of CNT/polymer composites are listed in Table 1. Despite the high thermal conductivity up to $\sim 10^{3} \mathrm{~W} / \mathrm{m}-\mathrm{K}$ of an individual CNT,[4] the thermal conductivity of composites with randomly dispersed CNTs in polymers is typically smaller than $1 \mathrm{~W} / \mathrm{m}-\mathrm{K}$ even with CNT concentrations up to $80 \mathrm{wt} \%$.[32-38] The low macroscopic thermal conductivity of the CNT-polymer composites can be attributed to thermal contact resistance at CNT-CNT 
junctions. $[9,12,31]$ On the other hand, composites made of aligned CNTs have higher thermal conductivity values, $[17,18,39]$ which confirms that thermal transport through fewer CNT-CNT junctions is much more effective than that of polymer composites containing randomly dispersed CNTs. However the high mechanical strength of aligned CNTs along the axial direction hinders accommodating the surface asperity, limiting the improvement of thermal contact resistance at the interface between TIMs and heat sources/sinks.[40, 41] It is well known that CNT has strong mechanical property with a high Young's modulus of $\sim 1 \mathrm{TPa},[42]$ which is extremely hard to deform along the axial direction.

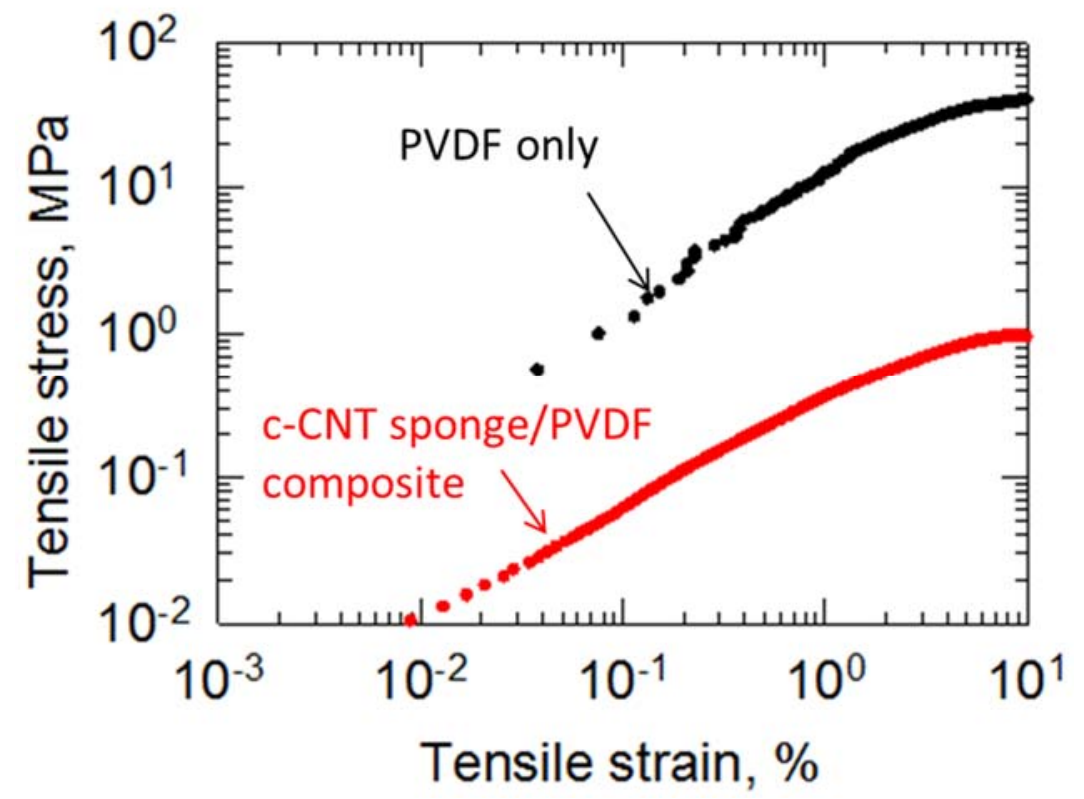

Figure 3. Stress-strain curves for a c-CNT composite (red dots) and PVDF only (black dots).

Hence the mechanical properties of the c-CNT composite and PVDF only were measured using a dynamic mechanical analysis instrument (Mettler Toledo TT-DMA), as shown in Fig. 3. 
It was found that the Young's modulus (50 MPa) of the composite was much lower than $1.5 \mathrm{GPa}$ for PVDF only and previously reported CNT-filled composites with various polymers such as polyepoxy (3.7 GPa for polyepoxy only; $4.2 \mathrm{GPa}$ for polyexpoxy/CNT composite with $0.15 \mathrm{wt} \%$ $\mathrm{CNT}),[43]$ polycarbonate $(2.3 \mathrm{GPa}$ for polycarbonate only; $4.1 \mathrm{GPa}$ for polycarbonate/CNT composite with $8 \mathrm{wt} \% \mathrm{CNT}),[44]$ and poly(vinyl alcohol) (1.2 GPa for poly(vinyl alcohol) only; 2.4 GPa for poly(vinyl alcohol)/CNT composite with $0.2 \mathrm{wt} \% \mathrm{CNT}$ ).[45] When CNT is added, polymer modulus values typically increases. At high strain, our composite showed two orders of magnitude lower stress values (Fig. 3) compared to the PVDF only sample, suggesting large deformability of our composite, which is essential to accommodate high surface asperity. In general, low modulus and large deformability indicate poor heat conduction since phonon transport through weak bonding is retarded. Here our composites consist of flexible porous sponge structures whose junctions are covalently bonded, and thereby high thermal conductivity can be maintained even with the small modulus and the large deformability. Additionally, our c-CNT composite showed a low thermal expansion coefficient (Fig. S5).

\section{Conclusions}

In summary, we have fabricated a new type elastomeric TIMs by infiltrating PVDF into CNT sponges using facile, scalable, and low-cost synthesis processes, which makes our composites very attractive as TIM. CNTs in the sponge structure were covalently bonded, resulting in a low Young's modulus but our composite maintained a decent thermal conductivity.

To the best of our knowledge, our experimental study on forming covalent bonding at CNT-CNT 
junctions in 3D bulks to avoid a large thermal contact resistance between CNTs is reported for the first time. Further study to control the density of covalently bonded CNT junctions may provide us to find optimal conditions for desired thermal and mechanical properties. This strategy makes it possible to obtain both high deformability and high thermal conductivity, which are difficult to have simultaneously due to their adverse correlation.

\section{References}

[1] Park W, Choi K, Lafdi K, Yu C. Influence of Nanomaterials in Polymer Composites on Thermal Conductivity. J Heat Transfer. 2012;134(4):041302-.

[2] Narumanchi S, Mihalic M, Kelly K, Eesley G. Thermal interface materials for power electronics applications. ITHERM, IEEE. 2008:395-404.

[3] Uetani K, Ata S, Tomonoh S, Yamada T, Yumura M, Hata K. Elastomeric Thermal Interface Materials with High Through-Plane Thermal Conductivity from Carbon Fiber Fillers Vertically Aligned by Electrostatic Flocking. Adv Mater. 2014;26(33):5857-62.

[4] Yu C, Shi L, Yao Z, Li D, Majumdar A. Thermal Conductance and Thermopower of an Individual Single-Wall Carbon Nanotube. Nano Lett. 2005;5(9):1842-6.

[5] Kim D, Kim Y, Choi K, Grunlan JC, Yu C. Improved Thermoelectric Behavior of Nanotube-Filled Polymer Composites with Poly(3,4-ethylenedioxythiophene) Poly(styrenesulfonate). ACS Nano. 2010;4:513-23.

[6] Yu C, Choi K, Yin L, Grunlan JC. Light-Weight Flexible Carbon Nanotube Based Organic Composites with Large Thermoelectric Power Factors. ACS Nano. 2011;5:7885-92. 
[7] Yu C, Kim YS, Kim D, Grunlan JC. Thermoelectric behavior of segregated-network polymer nanocomposites. Nano Lett. 2008;8:4428.

[8] Wang H, Hsu J-H, Yi S-I, Kim SL, Choi K, Yang G, et al. Thermally Driven Large N-Type Voltage Responses from Hybrids of Carbon Nanotubes and Poly(3,4-ethylenedioxythiophene) with Tetrakis(dimethylamino)ethylene. Adv Mater. 2015;27(43):6855-61.

[9] Yang J, Yang Y, Waltermire SW, Gutu T, Zinn AA, Xu TT, et al. Measurement of the Intrinsic Thermal Conductivity of a Multiwalled Carbon Nanotube and Its Contact Thermal Resistance with the Substrate. Small. 2011;7(16):2334-40.

[10] Hone J, Whitney M, Piskoti C, Zettl A. Thermal conductivity of single-walled carbon nanotubes. Phys Rev B. 1999;59(4):R2514-R6.

[11] Yang J, Waltermire S, Chen Y, Zinn AA, Xu TT, Li D. Contact thermal resistance between individual multiwall carbon nanotubes. Appl Phys Lett. 2010;96(2):023109.

[12] Yu C, Saha S, Zhou JH, Shi L, Cassell AM, Cruden BA, et al. Thermal contact resistance and thermal conductivity of a carbon nanofiber. J Heat Transfer. 2006;128(3):234-9.

[13] Jang I, Sinnott SB, Danailov D, Keblinski P. Molecular Dynamics Simulation Study of Carbon Nanotube Welding under Electron Beam Irradiation. Nano Lett. 2004;4(1):109-14.

[14] Banhart F. The Formation of a Connection between Carbon Nanotubes in an Electron Beam. Nano Lett. 2001;1(6):329-32.

[15] Meng FY, Shi SQ, Xu DS, Yang R. Size effect of X-shaped carbon nanotube junctions. Carbon. 2006;44(7):1263-6. 
[16] Yang X, Chen D, Han Z, Ma X, To AC. Effects of welding on thermal conductivity of randomly oriented carbon nanotube networks. Int J Heat Mass Transfer. 2014;70:803-10.

[17] Lin W, Moon K-S, Wong CP. A Combined Process of In Situ Functionalization and Microwave Treatment to Achieve Ultrasmall Thermal Expansion of Aligned Carbon Nanotube-Polymer Nanocomposites: Toward Applications as Thermal Interface Materials. Adv Mater. 2009;21(23):2421-4.

[18] Marconnet AM, Yamamoto N, Panzer MA, Wardle BL, Goodson KE. Thermal Conduction in Aligned Carbon Nanotube-Polymer Nanocomposites with High Packing Density. ACS Nano. 2011;5(6):4818-25.

[19] Wang M, Li T, Yao Y, Lu H, Li Q, Chen M, et al. Wafer-Scale Transfer of Vertically Aligned Carbon Nanotube Arrays. J Am Chem Soc. 2014;136(52):18156-62.

[20] Shahil KMF, Balandin AA. Graphene-Multilayer Graphene Nanocomposites as Highly Efficient Thermal Interface Materials. Nano Lett. 2012;12(2):861-7.

[21] Ohashi M, Kawakami S, Yokogawa Y, Lai G-C. Spherical Aluminum Nitride Fillers for Heat-Conducting Plastic Packages. J Am Ceram Soc. 2005;88(9):2615-8.

[22] Nikkeshi S, Kudo M, Masuko T. Dynamic viscoelastic properties and thermal properties of Ni powder-epoxy resin composites. J Appl Polym Sci. 1998;69(13):2593-8.

[23] Yang G, Choi W, Pu X, Yu C. Scalable synthesis of bi-functional high-performance carbon nanotube sponge catalysts and electrodes with optimum C-N-Fe coordination for oxygen reduction reaction. Energ Environ Sci. 2015;8(6):1799-807.

[24] Najafi E, Kim J-Y, Han S-H, Shin K. UV-ozone treatment of multi-walled carbon 
nanotubes for enhanced organic solvent dispersion. Colloids Surf A. 2006;284-285:373-8.

[25] Pradhan NR, Duan H, Liang J, Iannacchione GS. The specific heat and effective thermal conductivity of composites containing single-wall and multi-wall carbon nanotubes. Nanotechnology. 2009;20(24):245705.

[26] Navid A, Lynch CS, Pilon L. Purified and porous poly(vinylidene fluoride-trifluoroethylene) thin films for pyroelectric infrared sensing and energy harvesting. Smart Mater Struct. 2010;19(5):055006.

[27] Lehman JH, Terrones M, Mansfield E, Hurst KE, Meunier V. Evaluating the characteristics of multiwall carbon nanotubes. Carbon. 2011;49(8):2581-602.

[28] Springer GS, Tsai SW. Thermal Conductivities of Unidirectional Materials. J Compos Mater. 1967;1(2):166-73.

[29] Yu J, Qian R, Jiang P. Enhanced thermal conductivity for PVDF composites with a hybrid functionalized graphene sheet-nanodiamond filler. Fibers Polym. 2013;14(8):1317-23.

[30] Li Y, Huang X, Hu Z, Jiang P, Li S, Tanaka T. Large Dielectric Constant and High Thermal Conductivity in Poly(vinylidene fluoride)/Barium Titanate/Silicon Carbide Three-Phase Nanocomposites. ACS Appl Mater Interfaces. 2011;3(11):4396-403.

[31] Prasher RS, Hu XJ, Chalopin Y, Mingo N, Lofgreen K, Volz S, et al. Turning Carbon Nanotubes from Exceptional Heat Conductors into Insulators. Phys Rev Lett. 2009;102(10):105901.

[32] Meng C, Liu C, Fan S. A Promising Approach to Enhanced Thermoelectric Properties Using Carbon Nanotube Networks. Adv Mater (Weinheim, Ger). 2010;22(4):535-9. 
[33] Yu A, Itkis ME, Bekyarova E, Haddon RC. Effect of single-walled carbon nanotube purity on the thermal conductivity of carbon nanotube-based composites. Appl Phys Lett. 2006;89(13):133102.

[34] Du F, Guthy C, Kashiwagi T, Fischer JE, Winey KI. An infiltration method for preparing single-wall nanotube/epoxy composites with improved thermal conductivity. J Polym Sci, Part B: Polym Phys. 2006;44(10):1513-9.

[35] Guthy C, Du F, Brand S, Winey KI, Fischer JE. Thermal Conductivity of Single-Walled Carbon Nanotube/PMMA Nanocomposites. J Heat Transfer. 2007;129(8):1096-9.

[36] Bonnet P, Sireude D, Garnier B, Chauvet O. Thermal properties and percolation in carbon nanotube-polymer composites. Appl Phys Lett. 2007;91(20):201910.

[37] Jakubinek MB, White MA, Mu M, Winey KI. Temperature dependence of thermal conductivity enhancement in single-walled carbon nanotube/polystyrene composites. Appl Phys Lett. 2010;96(8):083105.

[38] Gojny FH, Wichmann MHG, Fiedler B, Kinloch IA, Bauhofer W, Windle AH, et al. Evaluation and identification of electrical and thermal conduction mechanisms in carbon nanotube/epoxy composites. Polymer. 2006;47(6):2036-45.

[39] Huang H, Liu CH, Wu Y, Fan S. Aligned Carbon Nanotube Composite Films for Thermal Management. Adv Mater. 2005;17(13):1652-6.

[40] Mecklenburg M, Mizushima D, Ohtake N, Bauhofer W, Fiedler B, Schulte K. On the manufacturing and electrical and mechanical properties of ultra-high wt.\% fraction aligned MWCNT and randomly oriented CNT epoxy composites. Carbon. 2015;91:275-90. 
[41] Nam TH, Goto K, Yamaguchi Y, Premalal EVA, Shimamura Y, Inoue Y, et al. Effects of CNT diameter on mechanical properties of aligned CNT sheets and composites. Composites Part A. 2015;76:289-98.

[42] Treacy MMJ, Ebbesen TW, Gibson JM. Exceptionally high Young's modulus observed for individual carbon nanotubes. Nature. 1996;381(6584):678-80.

[43] Leon V, Parret R, Almairac R, Alvarez L, Babaa MR, Doyle BP, et al. Spectroscopic study of double-walled carbon nanotube functionalization for preparation of carbon nanotube / epoxy composites. Carbon. 2012;50(14):4987-94.

[44] Chen L, Pang X-J, Yu Z-L. Study on polycarbonate/multi-walled carbon nanotubes composite produced by melt processing. Mater Sci Eng A. 2007;457(1-2):287-91.

[45] Deng L, Eichhorn SJ, Kao C-C, Young RJ. The Effective Young's Modulus of Carbon Nanotubes in Composites. ACS Appl Mater Interfaces. 2011;3(2):433-40. 